\title{
Stretched exponential decay of Majorana edge modes in many-body localized Kitaev chains under dissipation
}

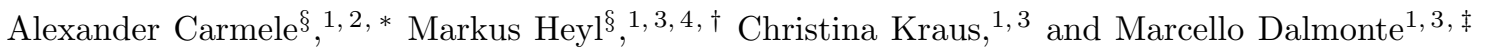 \\ ${ }^{1}$ Institute for Quantum Optics and Quantum Information of the Austrian Academy of Sciences, A-6020 Innsbruck, Austria \\ ${ }^{2}$ Institut für Theoretische Physik, Technische Universität Berlin, Hardenbergstraße 36, 10623 Berlin, Germany \\ ${ }^{3}$ Institute for Theoretical Physics, University of Innsbruck, A-6020 Innsbruck, Austria \\ ${ }^{4}$ Physik Department, Technische Universität München, 85747 Garching, Germany
}

\begin{abstract}
We investigate the resilience of symmetry-protected topological edge states at the boundaries of Kitaev chains in the presence of a bath which explicitly introduces symmetry-breaking terms. Specifically, we focus on single-particle losses and gains, violating the protecting parity symmetry, which could generically occur in realistic scenarios. For homogeneous systems, we show that the Majorana mode decays exponentially fast. By the inclusion of strong disorder, where the closed system enters a many-body localized phase, we find that the Majorana mode can be stabilized substantially. The decay of the Majorana converts into a stretched exponential form for particle losses or gains occuring in the bulk. In particular, for pure loss dynamics we find a universal exponent $\alpha \simeq 2 / 3$. We show that this holds both in the Anderson and many-body localized regimes. Our results thus provide a first step to stabilize edge states even in the presence of symmetry-breaking environments.
\end{abstract}

PACS numbers: 03.65.Yz, 67.85.Lm, 71.10.Pm

\section{INTRODUCTION}

Symmetry-protected topological states are a well established class of phases of matter which encompasses a rich variety of microscopic incarnations, ranging from spin chains to fermionic systems ${ }^{1+3}$. These phases of matter are a direct manifestation of a (non-local) symmetry in a model Hamiltonian, first identified in the context of the Haldane phase of spin-1 chains ${ }^{4}$, which can give rise to topological order and zero energy modes at the sample boundaries. A prominent example of this mechanism is provided by the Kitaev chain 5 which exhibits Majorana edge states ${ }^{6}$ once defined on a open geometry. These edge modes, which have anyonic statistics and can be potentially useful for quantum information purposes $^{7}$, have a direct relation with a global $\mathbb{Z}_{2}$ parity symmetry of certain one-dimensional superconductors ${ }^{5}$, usually granted by the proximity effect. However, naturally occurring dissipation can explicitly break the protecting microscopic symmetry 819 . In this context the major challenge is to stabilize the Majorana modes by exploiting additional mechanisms which could counteract the drastic action of symmetry breaking terms in open quantum system settings.

In this work, we investigate the resilience of Majorana edge modes in the presence of incoherent symmetrybreaking dissipation. We show that strong disorder can lead to an exponential gain in the edge mode's stability compared to the case of a homogeneous system. While in a homogeneous system we show that the temporal decay is exponential, in presence of disorder we find that the Majorana mode occupation decays in a stretched exponential form. Remarkably, when the coupling to the environment induces pure particle losses, the associated exponent $\alpha \simeq 2 / 3$ is universal and independent of any microscopic details within our numerical accuracy.
The effect of disorder on the time-dependent dynamics of closed quantum many-body systems has been actively investigated in both non-interacting and interacting scenarios. For noninteracting particles in low dimensions, disorder leads to Anderson localization (AL): all single-particle eigenstates become localized immediately for infinitesimal disorder 10111 . Recently, the concept of AL has been generalized to interacting systems in the context of many-body localization (MBL) 12 15. Importantly, compared to quantum many-body systems in thermodynamic phases, MBL systems display unconventional properties 14 17. In particular, this encompasses the possibility of phase transitions at nonzero temperature in one dimension $13[18$ 20. Moreover, it has been shown that topological properties, in one-dimensional thermodynamic systems only present in ground states, can survive over all of the spectrum 21,23 , greatly enhancing the regime of stability for topological order in closed systems 24 .

Motivated by these findings, we address in this work the fundamental question if and how the MBL mechanism can affect the stability of topological order, and in particular the resilience of edge modes, when the system is coupled to an incoherent bath. This question finds natural applications in diverse systems, such as solid state devices and cold atom settings, which have inherent sources of dissipation. Our results support the conclusion that MBL qualitatively enhances the resilience of edge states in symmetry-protected topological states even in the presence of the worst-case scenario of a bath inducing symmetry-breaking perturbations.

In concrete, we study the combined effects of disorder and interactions in a Kitaev chain coupled to a Markovian bath which explicitly breaks the parity symmetry of the system. The setup we have in mind is portrayed in Fig. 1. and is described in detail in Sec. II. Our analysis 
focuses on the decay of the Majorana edge mode under the effect of particle losses, starting from an initial state where such mode is occupied. In Sec. III] we first of all study the case of a homogeneous system as a benchmark, where we show that the Majorana mode decays exponentially as a function of time. Specifically, we consider a particular setup where the dissipation acts only on a part of the system (red area in Fig. 1) with a varying region near the boundaries that is not coupled to the Markovian bath. This represents an idealized model system which, however, is potentially relevant for a variety of experimental systems as we discuss in detail in Sec. III Both in the presence and in the absence of interactions, we find that for the case of particle losses the survival time of the Majorana edge mode increases exponentially with the number of protected sites, that is, the number of sites close to the edge where no dissipation occurs. This increased lifetime can be immediately traced back to the nature of the edge mode wave function which only exhibits an exponential tail extending into the bulk.

In Sec. IV] and Sec. V] we discuss how disorder drastically affects the above mentioned scenario. As anticipated before, in contrast to the exponential decay in the homogeneous system, the Majorana edge mode decays as a stretched exponential with a universal exponent $\alpha \simeq 2 / 3$ for the pure loss dynamics. This points towards an increased robustness of the edge mode when compared to the clean case. In particular, this universal behavior occurs both in the Anderson and many-body localized regimes, i.e., also in the presence of interactions which makes the system generic. This shows that disorder, and, in general, localization effects, which induce non-ergodic dynamics in the system, are not detrimental to the survival of Majorana modes in the presence of a bath, and on the contrary, greatly enhance their stability. This identifies disorder as a key mechanism to stabilize topological features in open quantum systems, and thus to systematically improve the stability of potential quantum memories even in the presence of dissipation which explicitly breaks the symmetry protecting topological order. Finally, in Sec. V] we present a case study for the loss and gain case, where a qualitatively similar behavior can be observed as well. We find that the decay of the Majorana again is of stretched exponential form.

\section{MODEL HAMILTONIAN AND MASTER EQUATION}

The Kitaev chain can be realized in a variety of different experimental architectures including AMO as well as solid-state setups 6/25]35/36) 39 . Its Hamiltonian describes spinless fermions on a lattice of $\mathrm{N}$ sites with a pairing term ${ }^{5}$ :

$$
H_{\mathrm{K}}=-\sum_{l=1}^{N-1}\left[\left(J_{l} c_{l}^{\dagger} c_{l+1}-\Delta_{l} c_{l} c_{l+1}\right)+\text { h.c. }\right]+\mu \sum_{l=1}^{N} c_{l}^{\dagger} c_{l},
$$

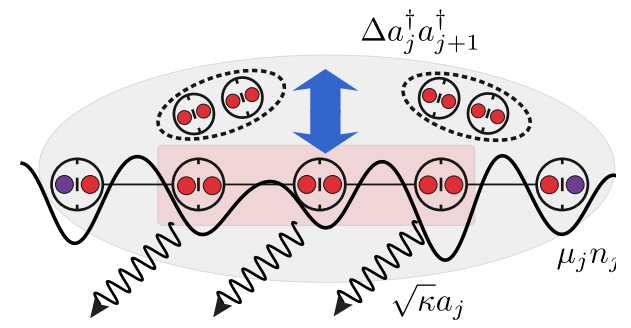

Figure 1. (color online) Schematic picture of the dynamics of a disordered Kitaev chain subject to a bath. The system is initialized in the ground state of the ideal Kitaev chain, where it is better visualized splitting the fermionic degree of freedom at each site into a pair of Majorana fermions (small full circles). In the ground state, all Majorana fermions in the middle couple over bonds, leaving the first and last Majorana fermion uncoupled (blue circled). The dynamics is the subject to a disordered potential (black, thick line) in addition to pairing to a superconductor (grey shaded area) and to the action of a bath acting on the bulk (red area). The presence of the bath explicitly breaks the parity symmetry of the wire due to particle loss.

where $c_{l}^{\dagger}, c_{l}$ are fermionic creation/annihilation operators at site $l=1, \ldots, N$. The first two terms represent tunneling between lattice sites as well as pairing induced by a superconductor, whose magnitude can, in principle, depend on the specific lattice site, and the last term is a chemical potential. In the homogeneous regime $J_{l}=J, \Delta_{l}=\Delta,|\mu / J|<1, \Delta \neq 0$, the model hosts a symmetry-protected topological phase directly related to the exact parity symmetry of the Hamiltonian, that is, $[H, P]=0, P=\prod_{l=1}^{N}\left(1-2 n_{l}\right)$, where $n_{l}=c_{l}^{\dagger} c_{l}$ is the local particle number operator. In order to assure that the properties of the system are generic we account for a weak, parity-preserving nearest-neighbor interaction making the system non-integrable ${ }^{40}$. This yields as the full Hamiltonian:

$$
H=H_{\mathrm{K}}+V, \quad V=U \sum_{l=1}^{N-1}\left[n_{l}-\frac{1}{2}\right]\left[n_{l+1}-\frac{1}{2}\right],
$$

with $U / J \ll 1$. Notice that this model can be mapped via a Jordan-Wigner transformation to a transverse-field $\mathrm{XYZ}$ chain, and as such is nonintegrable and generi ${ }^{41}$. In the case where $\mu=U=0, J=\Delta$, the ground state (GS) of the open Kitaev chain supports a localized Majorana edge mode (see illustration in Fig. 1) whose wave function is restricted to the edge lattice sites only ${ }^{\sqrt{5}}$. In the odd parity sector for odd number of sites, the GS reads $\underline{42}$

$$
\left|\psi_{0}\right\rangle=2^{-\frac{N}{2}}\left[\otimes_{i=1}^{N}\left(|1\rangle_{i}+|0\rangle_{i}\right)+\otimes_{i=1}^{N}\left(|1\rangle_{i}-|0\rangle_{i}\right)\right] .
$$

where the occupation of the zero energy mode, the edgeedge correlation, $\theta$ satisfies:

$$
\theta=\left\langle\psi_{0}|\Theta| \psi_{0}\right\rangle=1, \quad \Theta=i\left(c_{1}+c_{1}^{\dagger}\right)\left(c_{N}-c_{N}^{\dagger}\right) .
$$


and $\Theta$ denotes the Majorana mode occupation operator. If we would slightly perturb our Hamiltonian with $\mu, U>$ 0 , but still sufficiently small not to leave the topological phase, the Majorana zero mode develops an exponential tail extending into the bulk, with most of its weight still located at the boundaries of the chain 43144 .

The above particular choice of $\left|\psi_{0}\right\rangle$ represents an idealized initial condition which, of course, relies on an exact implementation of the Hamiltonian, or on a perfect dissipative state preparation of the state of interest (along the lines of Ref. 36). As we would like to argue at the end of this section, however, our full setup is still sufficiently general in order to describe the generic dynamics.

Remarkably, it has been recently shown how the topological features of the ground state in one-dimensional systems - in particular, the presence of edge modes and a degenerate entanglement spectrum - can be extended to the entire spectrum in the presence of strong disorder $2123|24| 45]$. This mechanism, a direct consequence of many-body localization, can lead to a stabilization of topological features at nonzero energy density above the ground state in a closed system.

Our main focus here is to investigate the fate of the Majorana zero mode in the presence of symmetry- breaking dissipation. The microscopic details of the particle loss and/or gain mechanism will strongly depend on the experimental architecture. In a cold atom experiment, losses are naturally occurring as either inelastic scattering processes due to the immersion in a BEC of molecules, or background collisions 46147 . These effects are often very well modeled as a Markovian bath-48. In solid-state systems, loss and gain terms can emerge as a consequence of a coupling to a grand-canonical bath. For Kitaev chains realized by placing carbon nanotube wires on top of a p-wave superconductor, particle losses and gains can occur by electron tunneling into and from the substrate. In this work, we concentrate on the scenario where both the loss and gain can be described within a Master equation framework. At a qualitative level, this implies that the parity symmetry in the system is violated at all energy scales - which we interpret as a worst-case scenario for symmetry-protected topological states. The system dynamics can then be described using a Lindblad master equation of the form:

$$
\begin{aligned}
\partial_{t} \rho & =-\frac{i}{\hbar}[H, \rho]+\sum_{l=1}^{N} \kappa_{l}\left[c_{l} \rho c_{l}^{\dagger}-\frac{1}{2}\left\{c_{l}^{\dagger} c_{l}, \rho\right\}\right]+ \\
& +\sum_{l=1}^{N} \gamma_{l}\left[c_{l}^{\dagger} \rho c_{l}-\frac{1}{2}\left\{c_{l} c_{l}^{\dagger}, \rho\right\}\right],
\end{aligned}
$$

with $\rho$ the density matrix of the system, $\kappa_{l}$ and $\gamma_{l}$ are the local loss and gain rates, respectively. We consider uniform dissipation, but restricted to the bulk of the chain where $\kappa_{l}=\kappa, \gamma_{l}=\gamma$ for $d<l<N-d$, and $\kappa_{l}=\gamma_{l}=0$ otherwise with $d$ the distance of the first dissipative site from the edge. For an illustration, see Fig. 1. In other words, we mainly consider the influence of dissipation in the bulk, not at the boundaries.
The main motivation for the choice of the setup is twofold. First, from a theoretical point of view, by restricting dissipation to the bulk we address the fundamental question of whether information stored in Majorana modes is stable against symmetry-breaking processes in the bulk. While dissipation acting on the edges of the system opens a direct channel for the decay of the Majorana mode, protecting the boundary sites allows us to extract the bulk contributions and therefore to unravel the influence of the indirect channel. Second, our setup might be relevant also in a general experimental context where dissipation is not uniform and particle losses or gains are not occuring homogeneous throughout space. In that case, isolated regions with weak or even approximately vanishing decay rates can form for which our setup represents an idealized reduced model system.

We remark that we have checked via extensive numerical simulations whether the specific choice of the distribution of the decaying sites can have an impact on the dynamics and our main results. We found that solely the number of sites which are not affected by dissipation mattered in terms of the qualitative behavior - as long as the edge sites are not included.

The figure of merit in our study is the fate of the Majorana mode:

$$
\theta(t)=\operatorname{tr}[\rho(t) \Theta],
$$

as a function of time $t$ in presence of the incoherent bath. For our initial density matrix $\rho(t=0)=\left|\psi_{0}\right\rangle\left\langle\psi_{0}\right|$ with $\theta=1$, we numerically solve the master equation in Eq. (5). For the noninteracting system with $U=0$, the resulting master equation is of quadratic fermionic form and can therefore be solved efficiently for large systems 49150 . We use this noninteracting limit as a benchmark for our more general and exact numerical implementation where interactions can also be incorporated at the expense of limited system sizes. We find, however, that, for the considered scenarios, finite-size effects can be neglected (see discussion below). We start by computing the dynamics of $\theta(t)$ in the clean case, and then discuss the role of disorder, showing how the latter case displays to an exponential gain in stability of the Majorana mode when compare to the former one.

Let us note that $\theta$ in Eq. (6) does not measure the overlap with the idealized wave function of the Majorana mode with respect to the final Hamiltonian for the general parameter set considered in this work (the wave function will be generically localized at the edge, with a finite contribution in the bulk), but rather those contributions which are located right at the edges. This, however, is not a shortcoming as we will now shortly discuss. First of all, the dynamics at the edges has to reflect the dynamics of the full Majorana wave function because most of its weight is located right there. Moreover, we expect that the considered scenario is also the experimentally most relevant one. In particular, measuring operators located on one lattice site is much easier than measuring an extended object such as the full Majorana wave func- 

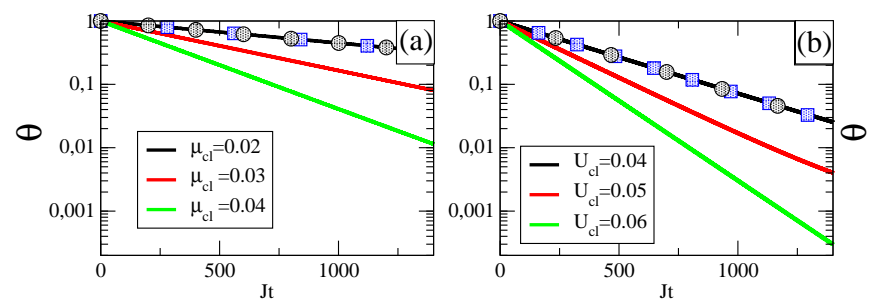

Figure 2. Impact of particle loss on the edge-edge correlation $\theta$ in the clean case $J_{l}=1$ : (a) with a finite external field $\mu=0.02,0.03,0.04(U=0)$ and $\kappa=1 / 2$ for one protected site $d=1$; (b) with an on-site repulsion $U=0.04,0.05,0.06(\mu=$ 0 ) and $\kappa=1$ for $d=1$. Circles and boxes in (a) and (b) show the dynamics for systems of different lengths $(N=9,11)$.

tion. In this context, it is also important to emphasize that the precise form of the Majorana wave function in a concrete experiment is not a priori known because it depends on a variety of experimental details - especially in the presence of interactions.

Finally, these arguments also motivate our specific choice of initial condition in Eq. (3). In any realistic experimental system it will, of course, not be possible to precisely prepare the considered state due to experimental imperfections. As long as the Majorana mode is initially occupied, however, our observable $\Theta$ is capable to detect it without knowledge about microscopic details. Therefore, we expect that these details only lead to quantitative and not qualitative changes.

\section{HOMOGENEOUS SYSTEM}

Using the model system described in the previous section we now aim at studying first the influence of symmetry-breaking particle losses for the homogeneous system. This will serve as a benchmark for the further analysis in Sec. IV where we will show that the Majorana edge mode can be stabilized by the inclusion of disorder. For the homogeneous system we analyze the noninteracting and interacting cases separately in order to single out the potential influence of integrability-breaking interactions.

The main result for the homogeneous system is that

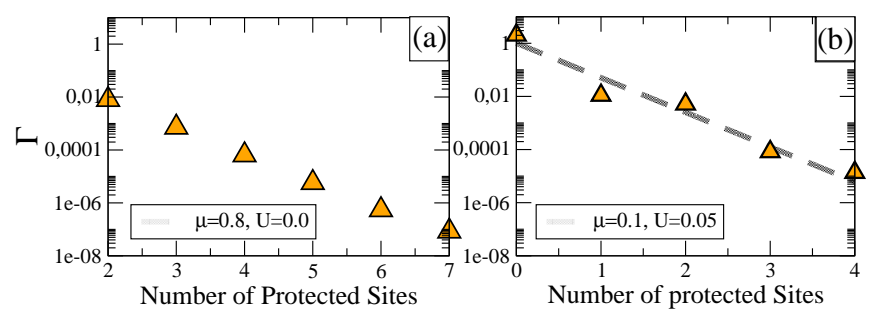

Figure 3. Panels (c-d): exponential dependence of the effective decay time $\Gamma$ on the number of protected sites $d$, with $\mathrm{N}$ $=43$ (11) in the left (right) panel. the Majorana mode occupation decays exponentially in time in consequence of the breaking of the protecting parity symmetry. However, we find that, remarkably, the associated decay rate $\Gamma$ is highly sensitive to the distance $d$ of the first dissipative site from the edge, but not to the microscopic parameters. In particular, interactions do not lead to a qualitative change of the dynamics but rather only increase the decay rates. Our main findings are summarized in Fig. 2 .

\section{A. Non-interacting case}

We start by considering the benchmark of noninteracting particles with $U=0$. In this limit we have used two different methods to solve the Master equation. On the one hand we have made use of the property that the Master equation at $U=0$ becomes a quadratic form in terms of fermionic operators. This allows us to use methods developed in Ref. 49, such that we are able to obtain the solution for large lattices up to $N=100$ sites. On the other hand we have solved the Master equation directly on the basis of a Runge-Kutta algorithm for up to $N=11$ lattice sites which can also be extended to interacting systems studied later on. By comparing the results of both methods, we have found that finite-size effects are negligible in the parameter regime considered here.

As anticipated before, we find that in the noninteracting homogeneous system the Majorana mode occupation $\theta(t)$ decays exponentially in time:

$$
\theta(t) \stackrel{t \rightarrow \infty}{\longrightarrow} e^{-\Gamma t},
$$

with $\Gamma$ the decay rate, both in the cases of a global bath acting on all sites, $\kappa_{l}=\kappa$, and in the case of a bath which does not act on a small region close to the edge, $\kappa_{l}=\kappa$ for $l \in[d+1, L-d]$ (with $d$ the number of protected sites close to the edge), and 0 otherwise. In the following, we focus on the bulk contribution, investigating the Majorana decay as a function of $d$.

In Fig. 2 a, time traces of the Majorana occupations are shown for the case where the first dissipating lattice site is located right next to the edge, i.e., $d=1$. As one can see, the decay rate depends crucially on the strength of the chemical potential. This can be traced back to a fundamental property of Kitaev chains at $\mu=0$. There the Majorana qubit decouples completely from the bulk chain and Majorana mode occupation operator $\Theta$ commutes with the Hamiltonian $\left[H_{\mathrm{K}}, \Theta\right]=0$. As a consequence, we have that $\theta(t)=1$ for all times $t$ when $\mu=0$. Therefore, at this particular point in parameter space the Majorana completely decouples from the dissipative dynamics and becomes completely stable. However, this stabilization mechanism depends crucially on parameter fine-tuning and any perturbation, always present in a realistic experiment, induces an exponential decay.

The infinitesimal sensitivity of the Majorana qubit stability can be seen by considering the effects of a non- 
vanishing chemical potential $\mu>0$, which is shown in Fig. 2 Then, the Majorana qubit experiences an effective coupling to the bulk modes and the exponential decay induced by the symmetry-breaking losses sets in. The decay rate grows with increasing chemical potential.

Remarkably, it is possible to increase the lifetime of the Majorana mode substantially also for the homogeneous system without parameter fine-tuning. Specifically, we find that the decay rate is exponentially sensitive to the distance $d$ of the edge from the first dissipative lattice site. This is shown in Fig. 2, where $\Gamma$ is plotted as a function of $d$. The decay rate is extracted numerically from two time points $t_{2}>t_{1}$ with $\Gamma=\log \left[\theta\left(t_{2}\right) / \theta\left(t_{1}\right)\right] /\left(t_{1}-t_{2}\right)$. We have checked that the resulting $\Gamma$ 's are independent of the precise choice of $t_{1}$ and $t_{2}$ as long as we are in the asymptotic long-time regime. As a consequence, in the case of pure particle losses, the bulk only contributes weakly to the decay of the Majorana. Notice that this is different from the case with losses and gain discussed in Sec. V. We attribute this strong sensitivity of the decay to the exponentially small overlap of the Majorana wave function with the loss mechanism on distant lattice sites. In particular, as long as the evolved Hamiltonian stays in the topological phase, the Majorana wave functions are exponentially localized in the vicinity of the edges. Therefore, the direct influence of a particle loss at a given lattice site on the Majorana mode is exponentially small. However, excitations that are created by a particle loss could, in principle, still propagate to the edges potentially inducing a decay of coherence. The numerical data, however, suggests that the dominant principle is solely the overlap of the Majorana wave function on the first dissipative lattice site.

\section{B. Interacting case}

After having discussed in detail the noninteracting homogeneous system we now aim at analyzing the influence of interactions. This is important for clarifying which of the effects are generic and independent of particular parameter choices. The numerical results for the interacting case are illustrated in Fig. 2, where, along the lines of the previous section, we check the resilience of the edge modes for increasing interaction strength.

As for the noninteracting case, we find that the Majorana edge modes decay exponentially in time, see the numerical data presented in Fig. 2. Again, the decay rate decreases exponentially with the distance $d$ of the first dissipating lattice site, see Fig. 3. In contrast to the noninteracting case, however, an even-odd effect of $\Gamma$ as a function of $d$ is visible. Importantly, the model is now generic which becomes apparent at the fine-tuned parameter point $\mu=0$ of the noninteracting model, see Sec. IIIA where the Majorana mode is stable for $d \geq 1$. In Fig. 22, we show time traces of $\theta(t)$ at $\mu=0$ but nonvanishing interactions. As one can see, interactions now lead to a decay of the edge mode.

Before we study the impact of disorder, we summarize the main result of this section: Symmetry-breaking perturbations lead to a decay of the Majorana qubit even if the edge states are protected from particle loss, independently on the presence or absence of interactions. However, the survival time of the edge mode increases exponentially with the number of protected sites $d$.

\section{MANY-BODY LOCALIZED KITAEV CHAIN}

For closed systems, it has been recently observed that disorder provides a generic mechanism to stabilize topological order throughout the full many-body spectrum, i.e., up to infinite temperature $23151 / 52$. Strikingly, this holds even for one-dimensional systems where long-range (topological) order at nonzero temperature is not possible in thermodynamic phases $13153 \sqrt[55]{5}$. These manybody localized systems are non-ergodic and consequently promise a key mechanism to stabilize qubits in closed quantum system settings 56 .

For Anderson-localized systems it is known that a coupling to low-temperature baths in general induces a nonzero conductance in the context of variable-range hopping 57 . However, depending on the spectral details of the bath, localization can persist in the sense that the coupling between system and bath becomes irrelevant at low energies 58 . For small baths, not of thermodynamic nature, it has been shown that localization can persist in a strong disorder limit $\underline{5960}$. In many-body localized systems the influence of low-temperature thermal baths onto the broadening of local spectra has been studied recently 14 . The static properties of MBL systems coupled to small baths and the transition of the combined system to a Wigner-Dyson statistics have been addressed in Ref. 61 .

In the following, we study the many-body localized Kitaev model in presence of an incoherent structureless bath described by the Master equation in Eq. (5). We show the stabilization property of many-body localized phases remarkably extends also to this worst-case open quantum system scenario, thus identifying nonergodicity of closed systems as a possible route to stabilize edge states in the presence of detrimental (symmetrybreaking) noise. Our main result, summarized in Fig. 4 . is that the Majorana mode can be stabilized by the inclusion of disorder with an exponential gain in resilience. Specifically, the exponential decay of the homogeneous system converts into a stretched exponential decay in presence of disorder, that is:

$$
\langle\theta(t)\rangle_{\mathrm{dis}} \stackrel{t \rightarrow \infty}{\longrightarrow} e^{-(\gamma t)^{\alpha}}
$$

with $\langle\ldots\rangle_{\text {dis }}$ denoting the average over disorder realizations. We find that within our numerical accuracy the associated exponent $\alpha$ is universal and close to

$$
\alpha \simeq 2 / 3
$$


In our numerical implementation, we choose bond disorder for simplicity by introducing inhomogeneous couplings $J_{l} \in[0, W], l=1 \ldots N-1$ sampled from independent uniform distributions of width $W=e J$. We will characterize the strength of the disorder via $J$ differing from $W$ by Euler's number $e$ such that the critical point of the noninteracting model is located at $\mu / J=1$ as in the clean case ${ }^{62163}$. In the following simulations, we are interested in the large disorder limit such that the localization length of most eigenstates is limited to few lattice spacings (thus limiting finite-size effects). For each parameter regime, the results are averaged over up to 10000 disorder realizations in order to ensure convergence. We expect, however, that the generic features are independent of this precise choice of bond disorder, in particular, because it is known that the properties of the bond-disordered Kitaev and the related Ising chain are equivalent to the case of site disorder.

Before diving into a detailed discussion of our results, let us comment on the case where the dissipative losses act on every lattice site, $\kappa_{l}=\kappa$, in particular, including the edges where the Majorana modes are located. In this case, we find that the Majorana mode occupation still decays exponentially as found for the homogeneous case. Thus, the Majorana qubit is not stable against incoherent onsite losses as one might expect. This local decoherence mechanism cannot be stabilized using disorder. However, as we will show below, protecting sites in the immediate vicinity of the boundaries lead to a substantial stabilization.

We start by discussing as a warm-up the noninteracting (Anderson) limit with nonzero chemical potential $\mu>0$ but $U=0$. Our numerical results on this case are shown in Fig. 4a. In panels 44-d, we plot the same quantity for a clean (c) and disordered system (d, same data as in a) as a function of the rescaled time $(J t)^{2 / 3}$. While in the clean system the decay stays exponential as discussed in the previous section (the curve bends down in lin-log scale), in the disordered case one gets an almost flat line (but for additional coherent oscillations), demonstrating an exponentially increased stability of the edge mode, and a decay according to $\alpha \simeq 2 / 3$. The decay becomes faster for larger chemical potential as this increases the coupling between the Majorana qubit and the bulk modes. An analysis of the time derivatives of the Majorana mode survival also confirms this decay.

The same stabilization mechanism emerges in the many-body localized regime, where the Majorana operator is expected to be renormalized, still be localized at the boundary 64 . The resulting decay of the Majorana mode for different interaction strength for the case $\mu=0$ is illustrated in Fig. 4 $\mathrm{b}$, f, while panels (e-d) show the comparison between clean and disordered case. Again, the decay is of the form of Eq. (8) with $\alpha \approx 2 / 3$.

Finally, let us consider the case of both nonzero interactions and nonzero chemical potentials. The resulting temporal behavior of the Majorana mode is shown in Fig. 5. Here we consider a comparable strength for
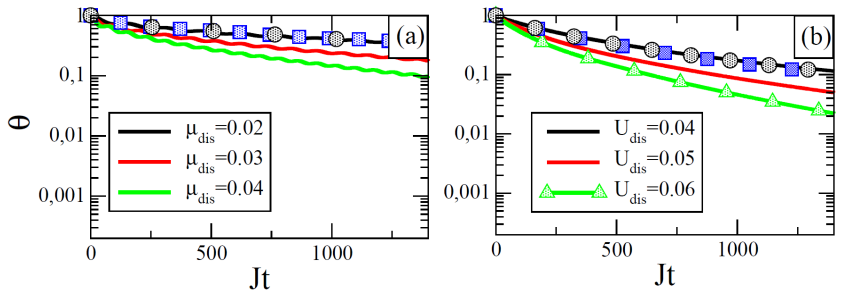

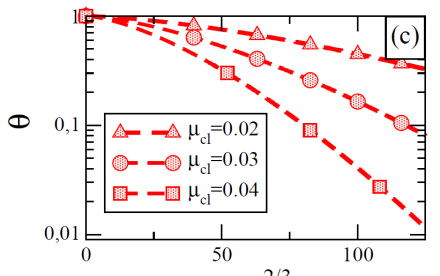

$(\mathrm{Jt})^{2 / 3}$

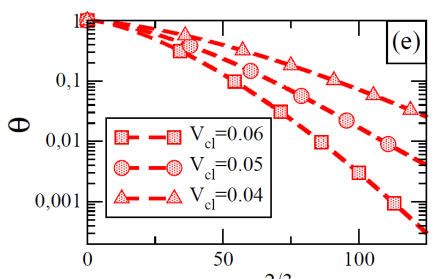

$(\mathrm{Jt})^{2 / 3}$

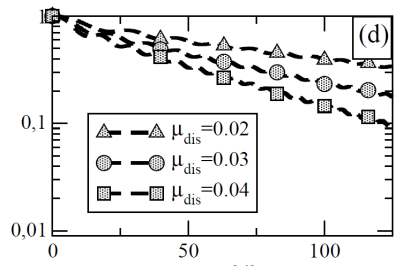

$(\mathrm{Jt})^{2 / 3}$

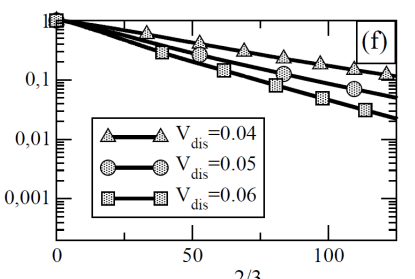

$(\mathrm{Jt})^{2 / 3}$
Figure 4. Impact of particle loss on the edge-edge correlation $\theta$ in the disordered case $J_{l} \in[0, e]:$ (a) with a finite external field $\mu=0.02,0.03,0.04(U=0)$ and $\kappa=1 / 2$ for one protected site $d=1$; (b) with an on-site repulsion $U=0.04,0.05,0.06(\mu=$ 0 ) and $\kappa=1$ for $d=1$. Circles and boxes in (a) and (b) show the dynamics for systems of different lengths $(\mathrm{N}=9,11)$. Panels (c-f) show the time trace with a scaled time-axis to illustrate $t^{2 / 3}$ dependence. In panels (c) and (e), we show the clean case, where the decay is exponential, and thus, in the rescaled axis, an algebraic decay with power larger than 1 is observed. In panels (d) and (f), we contrast this with the disordered case, where the decay is a stretched exponential with a power $\alpha \simeq 2 / 3$.

both $U=J / 5$ and $\mu=J / 5$ at a dissipation strength $\kappa / J=3 / 2$. For comparison also the respective homogeneous case is shown that exhibits the pure exponential decay already discussed in Sec. III. The many-body localized case on the other hand again shows the stretched exponential decay, with an additional coherent oscillation on top of the decay is found.

\section{EFFECTS OF PARTICLE GAIN}

So far, we concentrated on a setup where only particle losses occur. Such as scenario corresponds mainly to potential implementations based on cold atom setups, where the main dissipation channel is given by inelastic scattering between molecules in the reservoir, and particles in the wire. In a condensed-matter setting, a more natural environment would generically be a grand-canonical bath inducing both particle losses and 


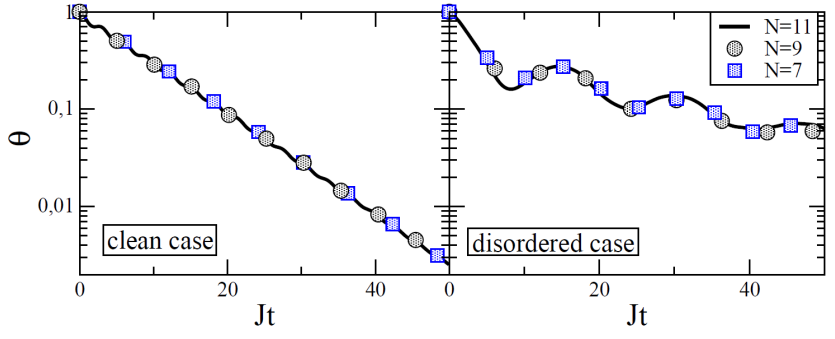

Figure 5. Limit of strong interaction and external field $\mu=$ $U=0.2$ with $\mathrm{d}=2$ protected sites and $\kappa=1.5$ for the clean and disordered case. Here, disorder increases the oscillation amplitude and slows down in order of magnitude the decay of the edge-edge correlation.

gains 65166. This is the scenario we focus on in this section. Specifically, we consider equal rates $\kappa_{l}=\gamma_{l}$ for both losses and gains for simplicity. Of course, baths in typical condensed-matter systems will not be exactly of the type described by the Master equation in Eq. (5) except maybe high-temperature baths. As anticipated in Sec. II], however, from the perspective of the stability of the Majorana mode, one can interpret the dynamics induced by this Master equation with incoherent losses and gains as a worst-case scenario.

We proceed as before by comparing the homogeneous case with the disordered case for a non-interacting, i.e. $U=0$, and interacting chain with $U \neq 0$. We will focus on a single protected site at each edge for simplicity. Our main results for this setup are summarized in Fig. 6. In principle, the overall picture remains identical compared to the case with pure losses. Again, we find that the presence of disorder suppresses the decoherence process of the Majorana mode. At first glance, also the qualitative picture is not changed, as we find a clear exponential decay in the clean case and a stretched exponential in the disordered case. This is not surprising, as from a symmetry point of view, both particle loss alone and particle loss and gain break the parity symmetry and in consequence lead to a loss of coherence of the Majorana edge mode.

However, apart from the first observation, that the additional particle gain does not completely change the overall impact of disorder on the decay of the Majorana, we find subtle modifications in the universality of the stretched exponential scaling. In contrast to previous case with pure losses where the exponent has been $\alpha \simeq 2 / 3$, see Eq. (8), we find a modified exponent $\alpha$ now in the presence of the additional particle gains. Specifically, from our numerical simulations the exponent turns out to be close to $\alpha \approx 3 / 4$. However, we have to emphasize that the situation is much less clear compared to the pure loss case. In particular, we find some slight dependence on the microscopic parameters. Therefore, we cannot conclude from our data that the exponent is universal in this case ${ }^{67}$ For many cases, we find a stretched exponential decay with approximately $\alpha=3 / 4$, but this
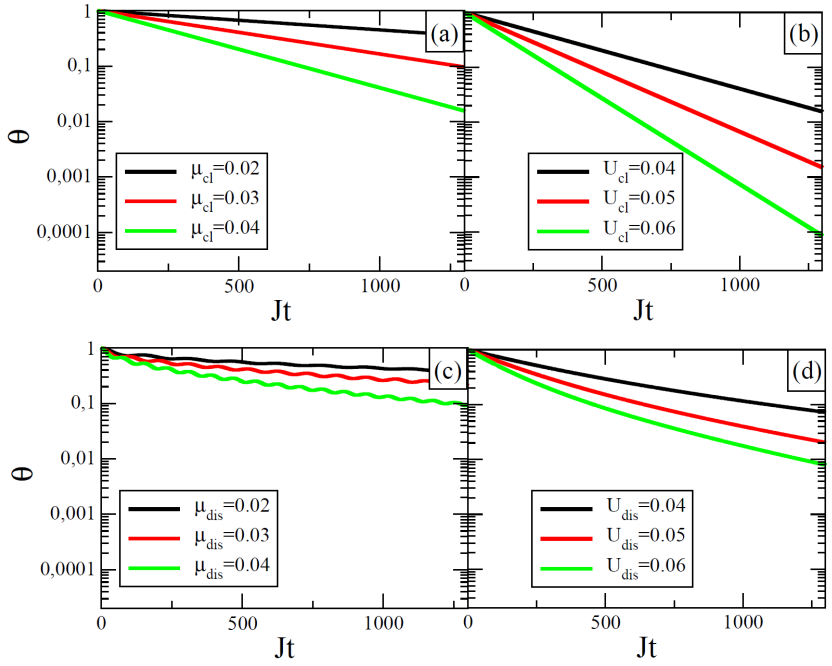

Figure 6. Impact of particle loss and gain on the edgeedge correlation $\theta$ in the clean (upper panel: a,b) and disordered case (lower panel: c,d) $J_{l} \in[0, e]$ : (a) with a finite external field $\mu=0.02,0.03,0.04(U=0)$ and $\kappa=1 / 2$ for one protected site $d=1$; (b) with an on-site repulsion $U=0.04,0.05,0.06(\mu=0)$ and $\kappa=1$ for $d=1$.

value can vary (for the parameters investigated) within a range $\pm 10 \%$.

Furthermore, it is worth mentioning that the balanced particle loss and gain leads to a more complex picture, compared to the pure loss scenario. For example, we observed partially decoupled bulk and edge dynamics in the case of an interacting chain of length $N=7$, where the distance exponential decay in the homogeneous case does not depend on the number of protected sites. This feature is a result of the uniform bulk dynamics. As soon as the bulk settles to a steady state due to gain and loss, the absolute length of the bulk is not of importance anymore. This stands in contrast to the inhomogeneous case, where such a distance dependence is tractable.

\section{CONCLUSIONS AND OUTLOOK}

We have presented a numerical study of the influence of a symmetry-breaking Markovian bath on the stability of Majorana edge modes in a Kitaev chain, including both disorder and interactions. In a clean setting, the occupation of the Majorana mode decays always exponentially as a function of time regardless of the system being interacting or not. The corresponding decay rate $\Gamma$ displays an exponential dependence on the number of sites close to the edge where dissipation is not acting, a direct consequence of the exponential localization of the mode wave-function in the pure loss case. This indicates that protecting even a very small number of sites can already improve the Majorana mode lifetime by several orders of magnitude with respect to the non-protected case, if gain is negligible or other interactions prevent an 
effectively reduced bulk length.

Strikingly, an even more solid protection mechanism is provided by the presence of disorder. In the closed system case without dissipation, many-body localization in disordered systems prevents thermalization even in the presence of interactions $\frac{14115}{}$. Importantly, it has been shown that topological properties can be present over the entire Hamiltonian spectrum in many-body localized systems $\sqrt{2324}$, and that bulk transport is severely inhibited in such phases - which generically display vanishing conductivity. We found that the consequences of these disorder-induced mechanisms upon the introduction of a Markovian bath are that the Majorana mode occupation decays in a qualitative different way than in the clean case. In the case of loss-only dissipation, which is directly relevant to cold atom systems, the decay form is a stretched exponential, with universal power $\simeq 2 / 3$, while in the case of both loss and gain - closer to solid state scenarios - the decay is still compatible with a stretched exponential, but with a larger power close to $\simeq 3 / 4$, whose determination is generically more challenging.

Overall, our results point toward the possibility of using many-body localization as a stabilizer mechanism of edge states in symmetry-protected topological states in the presence of a bath with explicitly breaks the symmetry. While the results presented herein are obtained using a controlled numerical approach, it would be of immediate relevance to develop a more general understanding using approximate solutions, which capture the main features of many-body localized systems coupled to baths, e.g., by exploiting the emergence of approximate integrals of motion, or by treating the bulk as an effective bath for the edges. Moreover, while the work here deals with symmetry-protected topological phases, the addition of disorder could serve as a stabilizing mechanism under dissipation even in phases supporting topological order in combination to long-range entanglement ${ }^{1 / 3}$, where it has been recently shown how disorder can be used to localize unwanted excitations ${ }^{68169}$, and could also help in further stabilizing Majorana modes against symmetrypreserving noise $e^{27 / 70}$.

\section{ACKNOWLEDGMENTS}

We acknowledge useful discussions with M. Baranov, E. Demler, M. Lukin, A. Scardicchio, and N. Yao, and we thank P. Zoller for fruitful criticism. Work in Innsbruck is partially supported by the ERC Synergy Grant UQUAM, SIQS, and SFB FoQuS (FWF Project No. F4016-N23). M. H. has been supported by the Deutsche Akademie der Naturforscher Leopoldina via Grant No. LPDS 2013-07 and LPDR 2015-01. A. C. acknowledges gratefully support from Alexander-von-Humboldt foundation through the Feodor-Lynen program.
* alex@itp.tu-berlin.de

† markus.heyl@uibk.ac.at

$\$$ These authors contributed equally to this work.

¥ marcello.dalmonte@uibk.ac.at

1 X.-G. Wen, Quantum Field Theory of Many-Body Systems (Oxford University Press, 2004).

2 A. M. Turner, F. Pollmann, and E. Berg, Phys. Rev. B 83, $075102(2011)$

${ }^{3}$ L. Fidkowski and A. Kitaev, Phys. Rev. B 81, 134509 (2010)

${ }^{4}$ T. Kennedy and H. Tasaki, Phys. Rev. B 45, 304 (1992)

5 A. Y. Kitaev, Physics-Uspekhi 44, 131 (2001).

${ }^{6}$ C. Beenakker, Annu. Rev. Con. Mat. Phys. 4, 113 (2013).

7 C. Nayak, S. H. Simon, A. Stern, M. Freedman, and S. D. Sarma, Rev. Mod. Phys. 80, 1083 (2008).

${ }^{8}$ L. Mazza, M. Rizzi, M. D. Lukin, and J. I. Cirac, Phys. Rev. B 88, 205142 (2013).

9 C. Bardyn, M. Baranov, C. Kraus, E. Rico, A. İmamoğlu, P. Zoller, and S. Diehl, New. J. Phys. 15, 085001 (2013).

10 P. W. Anderson, Phys. Rev. 109, 1492 (1958).

11 E. Abrahams, P. W. Anderson, D. C. Licciardello, and T. V. Ramakrishnan, Phys. Rev. Lett. 42, 673 (1979).

12 B. L. Altshuler, Y. Gefen, A. Kamenev, and L. S. Levitov, Phys. Rev. Lett. 78, 2803 (1997).

13 D. Basko, I. Aleiner, and B. Altshuler, Ann. of Phys. 321, 1126 (2006).

14 R. Nandkishore and D. A. Huse, Annu. Rev. Condens. Matter Phys. 6, 15 (2015).
15 E. Altman and R. Vosk, Annu. Rev. Condens. Matter Phys. 6, 383 (2015).

16 M. Znidaric, T. Prosen, and P. Prelovsek, Phys. Rev. B 77, 064426 (2008).

17 J. H. Bardarson, F. Pollmann, and G. Moore, Phys. Rev. Lett. 109, 017202 (2012).

18 I. L. Aleiner, B. Altshuler, and G. V. Shlyapnikov, Nature Phys. 6, 900 (2010).

19 V. Oganesyan and D. A. Huse, Phys. Rev. B 75, 155111 (2007).

20 A. Pal and D. A. Huse, Phys. Rev. B 82, 174411 (2010).

21 D. A. Huse, R. Nandkishore, V. Oganesyan, A. Pal, and S. L. Sondhi, Phys. Rev. B 88, 014206 (2013).

22 B. Bauer and C. Nayak, J. Stat. Mech. P09005 (2013).

23 A. Chandran, V. Khemani, C. R. Laumann, and S. L. Sondhi, Phys. Rev. B 89, 144201 (2014).

24 Y. Bahri, R. Vosk, E. Altman, and A. Vishwanath, (2013), arXiv:1307.4092v2

25 J. Alicea, Reports on Progress in Physics 75, 076501 (2012)

26 J. D. Sau and S. D. Sarma, Nat. Comm. 3, 964 (2012).

27 G. Goldstein and C. Chamon, Phys. Rev. B 84, 205109 (2011)

${ }_{28}$ R. M. Lutchyn, J. D. Sau, and S. D. Sarma, Phys. Rev. Lett. 105, 077001 (2010).

29 Y. Oreg, G. Refael, and F. V. Oppen, Phys. Rev. Lett. 105, $177002(2010)$

${ }^{30}$ V. Mourik, K. Zuo, S. M. Frolov, S. R. Plissard, E. P. A. M. Bakkers, and L. P. Kouwenhoven, Science 336, 
$1003(2012)$.

${ }^{31}$ L. P. Rokhinson, X. Liu, and J. K. Furdyna, Nat Phys 8, 795 (2012).

32 A. Das, Y. Ronen, Y. Most, Y. Oreg, M. Heiblum, and H. Shtrikman, Nat. Phys. 8, 887 (2012).

33 P. W. Brouwer, Science 336, 989 (2012)

34 S. Nadj-Perge, I. K. Drozdov, J. Li, H. Chen, S. Jeon, J. Seo, A. H. MacDonald, B. A. Bernevig, and A. Yazdani, Science 346, 602 (2014)

35 L. Jiang, T. Kitagawa, J. Alicea, A. R. Akhmerov, D. Pekker, G. Refael, J. I. Cirac, E. Demler, M. D. Lukin, and P. Zoller, Phys. Rev. Lett. 106, 220402 (2011).

36 S. Diehl, E. Rico, M. A. Baranov, and P. Zoller, Nature Physics 7, 971 (2011).

37 S. Nascimbéne, J. of Phys. B: Atomic, Molecular and Optical Physics 46, 134005 (2013).

38 J. C. Budich, S. Walter, and B. Trauzettel, Phys. Rev. B 85, 121405 (2012)

39 D. Rainis and D. Loss, Phys. Rev. B 85, 174533 (2012).

40 R. Steinigeweg and W. Brenig, arXiv:1312.4954 (2013).

41 A. Polkovnikov, K. Sengupta, A. Silva, and M. Vengalattore, Rev. Mod. Phys. 83, 863 (2011).

${ }^{42}$ We have checked that the qualitative behavior of the system is not affected by the initial state, unless the chain is initially empty.

43 P. W. Brouwer, M. Duckheim, A. Romito, and F. von Oppen, Phys. Rev. Lett. 107, 196804 (2011).

44 E. Stoudenmire, J. Alicea, O. A. Starykh, and M. P. Fisher, Phys. Rev. B 84, 014503 (2011).

45 S. Bravyi and R. König, Communications in Mathematical Physics 316, 641 (2012).

46 D. Jaksch and P. Zoller, Ann. of Phys. 315, 52 (2005).

47 J. R. Anglin and W. Ketterle, Nature 416, 211 (2002).

48 I. Bloch, J. Dalibard, and W. Zwerger, Rev. Mod. Phys. 80, 885 (2008), 0704.3011.

49 T. Prosen, New. J. Phys. 10, 043026 (2008).

50 S. Diehl, A. Micheli, A. Kantian, B. Kraus, H. Büchler, and P. Zoller, Nat. Phys. 4, 878 (2008).
51 J. Li, R.-L. Chu, J. K. Jain, and S.-Q. Shen, Phys. Rev. Lett. 102, 136806 (2009).

52 Q. Wu, L. Du, and V. E. Sacksteder, Phys. Rev. B 88, 045429 (2013).

53 M.-T. Rieder and P. W. Brouwer, Phys. Rev. B 90, 205404 (2014).

54 A. M. Lobos, R. M. Lutchyn, and S. Das Sarma, Phys. Rev. Lett. 109, 146403 (2012).

${ }^{55}$ R. Vosk and E. Altman, Phys. Rev. Lett. 110, 067204 (2013).

56 M. Franz, Nat. Nanotech. 8, 149 (2013).

57 N. F. Mott, Phil. Mag. 19, 835 (1969).

58 J. A. Hoyos and T. Vojta, Phys. Rev. B 85, 174403 (2012).

59 D. A. Huse, R. Nandkishore, F. Pietracaprina, V. Ros, and A. Scardicchio, arXiv:1412.7861 (2014).

${ }^{60}$ R. Nandkishore, arXiv:1506.05468 (2015).

61 S. Johri, R. Nandkishore, and R. N. Bhatt, Phys. Rev. Lett. 114, 117401 (2015).

62 P. Pfeuty, Phys. Lett. 72, 245 (1979).

63 D. S. Fisher, Phys. Rev. B 51, 6411 (1995).

${ }^{64}$ V. Ros, M. Mueller, and A. Scardicchio, Nucl. Phys. B 891, 420 (2015)

${ }^{65}$ Y. Su, D. Bimberg, A. Knorr, and A. Carmele, Phys. Rev. Lett. 110, 113604 (2013).

${ }^{66}$ G. Callsen, A. Carmele, G. Hönig, C. Kindel, J. Brunnmeier, M. Wagner, E. Stock, J. Reparaz, A. Schliwa, S. Reitzenstein, et al., Phys. Rev. B 87, 245314 (2013).

67 Whether the decaying exponent can become universal for larger systems cannot be addressed with the methods employed here.

${ }^{68}$ C. Stark, L. Pollet, A. Imamoğlu, and R. Renner, Phys. Rev. Lett. 107, 030504 (2011).

б9 J. R. Wootton and J. K. Pachos, Phys. Rev. Lett. 107, 030503 (2011)

${ }^{70}$ F. L. Pedrocchi and D. P. DiVincenzo, |arxiv.1505.03712

$71 \mathrm{Y} . \mathrm{Hu}$ and M. A. Baranov, |arxiv.1412.2547

${ }^{72} \mathrm{Y}$. Hu, Z. Cai, M. A. Baranov, and P. Zoller, |arxiv.1506.06977 